\title{
Perfluoroalkyl substances are associated with elevated blood pressure and hypertension in highly exposed young adults
}

Gisella Pitter ${ }^{1}$, Maryam Zare Jeddii ${ }^{2}$ Giulia Barbieri ${ }^{2}$, Massimo Gion ${ }^{3}$, Aline S. C. Fabricio ${ }^{3}$, Francesca Daprà ${ }^{4}$, Francesca Russo ${ }^{5}$, Tony Fletcher ${ }^{6}$ and Cristina Canova ${ }^{2}$ (D)

\begin{abstract}
Background: Residents in a large area of North-Eastern Italy were exposed to perfluoroalkyl substances (PFAS) via drinking water. Studies on the association between PFAS and blood pressure levels are limited, and results are inconsistent. Using cross-sectional data from the Regional health surveillance program, we aimed to quantify the associations between PFAS serum concentrations and blood pressure and hypertension prevalence.

Methods: The study comprised 16,224 individuals aged 20-39years. Pregnant women $(n=327)$, or individuals with missing information on the selected covariates $(n=111)$ were excluded, leaving 15,786 subjects for the analyses. Hypertension was defined as any self-reported diagnosis, use of antihypertensive drugs, or elevated systolic blood pressure $(\mathrm{SBP} \geq 140 \mathrm{mmHg}$ )/diastolic blood pressure (DBP $\geq 90 \mathrm{mmHg}$ ). Generalized additive models were used to investigate the relation between perfluorooctanoic acid (PFOA), perfluorooctane sulfonic acid (PFOS), perfluorohexane sulfonic acid (PFHXS), and perfluorononanoic acid (PFNA)) natural log (In) transformed and by decile, and SBP, DBP, hypertension, adjusted for potential confounders.
\end{abstract}

Results: Both SBP and DBP increased significantly with an increase in the In-transformed serum PFAS concentrations in a monotonic way. The predicted increase in SBP and DBP were $1.54 \mathrm{mmHg}(95 \% \mathrm{Cl} 0.61-2.47), 1.60 \mathrm{mmHg}(95 \% \mathrm{Cl}$ 0.92-2.27) from lowest to highest decile of PFOA. The associations were stronger for SBP in men and for DBP in women. One unit increase in each In-transformed PFAS was positively associated with an increased odd of hypertension in men: PFOA OR=1.06 (1.01-1.11), PFOS OR=1.13 (1.03-1.23), PFHxS OR=1.08 (1.02-1.15), PFNA OR= $1.20(1.02-1.40)$.

\footnotetext{
* Correspondence: cristina.canova@unipd.it

${ }^{2}$ Unit of Biostatistics, Epidemiology and Public Health, Department of

Cardio-Thoraco-Vascular Sciences and Public Health, Via Loredan 18, 35131

Padova, Italy

Full list of author information is available at the end of the article
}

(c) The Author(s). 2020 Open Access This article is licensed under a Creative Commons Attribution 4.0 International License, which permits use, sharing, adaptation, distribution and reproduction in any medium or format, as long as you give appropriate credit to the original author(s) and the source, provide a link to the Creative Commons licence, and indicate if changes were made. The images or other third party material in this article are included in the article's Creative Commons licence, unless indicated otherwise in a credit line to the material. If material is not included in the article's Creative Commons licence and your intended use is not permitted by statutory regulation or exceeds the permitted use, you will need to obtain permission directly from the copyright holder. To view a copy of this licence, visit http://creativecommons.org/licenses/by/4.0/ The Creative Commons Public Domain Dedication waiver (http://creativecommons.org/publicdomain/zero/1.0/) applies to the data made available in this article, unless otherwise stated in a credit line to the data. 


\begin{abstract}
(Continued from previous page)
Conclusions: Our findings suggest that serum PFAS concentrations were associated with increased systolic and diastolic blood pressure in a large highly exposed young adult population. Although the magnitude of the observed effect was relatively small, if confirmed it would be of public health relevance since even small increases in blood pressure levels at the population level may be associated to a raised risk of adverse outcomes such as cardiovascular disease and target organ damage.
\end{abstract}

Keywords: Perfluoroalkyl substances, Blood pressure, Hypertension, Epidemiology, Cross-sectional study, Community exposure

\section{Background}

Perfluoroalkyl substances (PFAS) are ubiquitous and highly persistent man-made chemicals widely used for a variety of commercial and industrial applications due to their grease-, stain, and water-repelling properties [1]. Consequently, human exposure to PFAS is widespread and mainly occurs through ingestion of contaminated food and dust [2]. Drinking water has been identified as a major source of exposure for many populations living near industrial and contaminated sites [3-6].

PFAS exposure has been associated with a number of risk factors of cardiovascular disease (CVD) including dyslipidemia, metabolic syndrome and thyroid disease [7]. High blood pressure is one of the most prevalent conditions increasing the risk of cardiovascular events [8]. Several studies in the general population [9-14], and just one on 500 highly exposed individuals in China [9] found positive associations between serum PFAS concentrations and hypertension. However, these findings were not demonstrated in other cross sectional studies [15-20], nor in two longitudinal studies on hypertension [21] and one on blood pressure trajectory [22]. The association of serum PFAS with blood pressure as a continuous outcome was evaluated in few cross-sectional studies, but with conflicting findings $[9,11,12,14]$.

Groundwater of a vast area of the Veneto Region (northeastern Italy) was found to be contaminated by PFAS from a manufacturing plant active since the late 1960s. Residents were exposed to high concentrations of PFAS, particularly perfluorooctanoic acid (PFOA) through drinking water, until autumn 2013 [6]. A publicly funded health surveillance program is established to aid in the prevention, early diagnosis, and treatment of chronic disorders possibly associated with PFAS exposure. Using cross-sectional data from the regional health surveillance program we were able to model concentration-response curves over a wide range of internal doses thanks to the high dispersion of serum PFOA concentrations and the large sample size, enable us to model concentration-response curves over a wide range of internal doses. Therefore, we aimed to quantify the associations between PFAS serum concentrations and blood pressure and hypertension prevalence in a large population of highly exposed young adults. Moreover, we systematically evaluated gender-specific associations.

\section{Methods}

\section{Participants and study design}

The study consisted of 16,224 individuals aged 20-39 years recruited in the health surveillance program offered to the community of Veneto Region who was exposed for several decades to PFAS via drinking water distributed by contaminated public waterworks. The health surveillance program has been described in more detail elsewhere [6]. In brief, the target population was initially constituted by people born between 1951 and 2002 and residing in the municipalities that were identified as in the area served by PFAS-contaminated waterworks. Surveillance involved the active invitation of the eligible population and the free offer of health examinations including: I) a questionnaire on personal health history and lifestyle habits, sociodemographic characteristics, self-reported height and weight; II) measurement of blood pressure; and III) nonfasting blood and urine samples.

Pregnant women $(n=327)$, and individuals with missing information on the selected covariates $(n=111)$ were excluded, leaving in the analyses 15,786 subjects. No missing data on exposure and outcome variables were present (See Supplementary Figure 1, Additional File 1).

\section{PFAS exposure}

Serum concentrations of twelve PFAS were measured by HPLC MS/MS (Shimadzu UFLC XR 20 Prominence coupled to Sciex API 4000): perfluorooctanesulfonate (PFOS), perfluorooctanoic acid (PFOA), perfluorohexanesulfonic acid (PFHxS), perfluorononanoic acid (PFNA), perfluoroheptanoic acid (PFHpA), perfluorobutanesulfonic acid (PFBS), perfluorohexanoic acid (PFHxA), perfluorobutanoic acid (PFBA), perfluoropentanoic acid (PFPeA), perfluorodecanoic acid (PFDeA), perfluoroundecanoic acid (PFUnA), and perfluorododecanoic acid (PFDoA). Details of the analytical method have been described elsewhere [6].

Method performances allow analytes to be detected as low as $0.1 \mathrm{ng} / \mathrm{mL}$ (LOD) and to be quantified above 0.5 $\mathrm{ng} / \mathrm{mL}$ (LOQ). Only four PFAS quantifiable in at least $40 \%$ of samples were considered for the analyses: PFOA (detected in $99.86 \%$ of people), PFOS (detected in 99.70\% of people), PFHxS (detected in $96.72 \%$ of people), PFNA (detected in $49.86 \%$ of people). Samples 
below the LOQ were assigned a value equal to LOQ $/ \sqrt{ } 2$. The most extreme outliers of PFAS serum levels were removed as followings: (PFOA $>700 \mathrm{mg} / \mathrm{L}(n=6)$, PFOS $>50 \mathrm{mg} / \mathrm{L}(n=9), \mathrm{PFHxS}>100 \mathrm{mg} / \mathrm{L}(n=3), \mathrm{PFNA}>10$ $\mathrm{mg} / \mathrm{L}(n=1))$.

\section{Blood pressure and hypertension}

Blood pressure (BP) was measured by trained nurses with participants first sitting at rest for at least five minutes, according to the European Society of Hypertension recommendations [23]. A validated semi-automatic sphygmomanometer with an appropriate cuff size for the arm circumference was used. When the first measure was $\geq 140 \mathrm{mmHg}$ for systolic blood pressure (SBP) or $\geq 90 \mathrm{mmHg}$ for diastolic blood pressure (DBP), a second measurement was taken at least two minutes apart. In general, 1714 subjects went through the second measurement. When the second measurement was within the cut-offs, the second measurement was used $(n=1078)$. Otherwise, the mean of the two measurements was considered when both measurements were above the cutoffs $(n=636)$.

Medical history data were collected directly from participants by trained nurses via structured software-based questionnaire using in-person interviews at the study enrolment. The questionnaire included items on personal health history ("Which diseases do you suffer from?") and medications ("Do you take any medication on a regular basis?" "If yes, which medications do you take?").

Hypertension was defined considering any selfreported diagnosis of hypertension, reported use of antihypertensive medications, or raised SBP $(\geq 140 \mathrm{mmHg})$ or DBP ( $\geq 90 \mathrm{mmHg})$.

\section{Covariates}

We obtained information on age, gender, country of birth, education level, smoking habits, body mass index (BMI), physical activity, history of certain diseases, medication, alcohol consumption, and food intakes including salt habit. Standard data checks and cleaning procedures (e.g. range and consistency checks) were used to minimize errors and missing values and to maximize data quality. Data on food consumption were transformed from number of serving per day/week/month to number of serving per week for all the food categories to create harmonized diet pattern classification. After checking the accuracy of data on BMI regarding height and weight, BMI was recalculated and classified as underweight $\left(<18.5 \mathrm{~kg} / \mathrm{m}^{2}\right)$, normal weight $(18.5-24.9$ $\left.\mathrm{kg} / \mathrm{m}^{2}\right)$, overweight $\left(25-29.9 \mathrm{~kg} / \mathrm{m}^{2}\right)$, obese $\left(\geq 30 \mathrm{~kg} / \mathrm{m}^{2}\right)$. Alcohol consumption was categorized in $0,1-2,3-6,7+$ alcohol units per week. Smoking status was subdivided into current smokers, previous smokers and nonsmokers. Degree of physical activity (Light, Moderate, or
Heavy) was defined based on an algorithm that combined information reported by the subject on intensity, duration, and frequency of all types of physical activity practiced during the week [6]. Countries of birth were classified in two categories based on geographical areas including: Italy plus other Highly Developed Countries, and High Migratory Pressure Countries. The time-lag between the beginning of the study (1st January 2017) and the date of enrollment was calculated for each subject and included as possible covariate (number of months). Information on the center in charge of the BP measurements was considered as possible confounder in statistical analyses.

Covariates to be included as potentially confounders of the BP/PFAS association were selected from the available variables, based on related literature, through the construction of a directed acyclic graph (DAG) representing the identification of a minimally sufficient set of variables to control confounding. The minimally sufficient adjustment set was identified using DAGitty v1.0 (www.dagitty.net) implemented in $\mathrm{R}$ ( $\mathrm{R}$ Development Core Team 2010, R Foundation for Statistical Computing, Vienna, Austria. ISBN 3-900,051-07-0, URL: http:// www.R-project.org/).

\section{Statistical analysis}

The serum concentrations of PFAS by gender were expressed as arithmetic mean, standard deviation (SD) and percentiles. Since data on PFAS were markedly skewed to the right, concentrations were natural $\log (\ln )$ transformed in order to improve normality of the data distribution. Spearman's correlation $(\rho)$ was used to describe pair-wise relations between the PFAS.

Our main outcomes are continuous SBP and DBP. For these analyses, participants with self-reported diagnosis of hypertension or under treatment with antihypertensive medications $(n=406)$ were excluded, leaving 15,380 subjects in analysis (See Supplementary Figure 1, Additional File 1).

We used generalized additive models (GAMs) to analyze the relation between each (ln) PFAS and BP outcomes, adjusted for the potential set of confounders. In order to explore the shape of possible associations between PFAS and BP levels the models used thin plate spline smooth terms [24] for the exposures and continuous covariates, and plotting the predicted values. Degree of smoothing was selected by generalized cross validation as implemented in the R package mgcv [25]. Since the spline analysis showed associations compatible with a linear relationship on the In PFAS, linear regression coefficient $(\beta)$ and $95 \%$ confidence intervals $(\mathrm{CI})$ were reported. Serum PFAS levels were also categorized into quartiles, in order to limit the influence of extreme values, with the exception of PFNA for which the large 
proportion below the LOQ did not allow the quartiles subdivision.

For the analyses on PFAS associations with hypertension prevalence, a binomial link function was used in the models and Odds Ratios (ORs) were calculated, together with their 95\% confidence intervals $(95 \% \mathrm{CI})$.

All analyses were fully adjusted for the established set of covariates: age, BMI, time-lag between the enrolment and the beginning of the study (all continuous variables modelled using thin plate spline) and categorical covariates including gender, physical activity, smoking habits, food consumption (tertiles or quartiles of fruit/vegetables, milk/yogurt, cheese, meat, sweet/snacks/sweet beverage, eggs, fish, bread/pasta/cereals per week), salt habit, country of birth, alcohol consumption, education level and center in charge of the BP measurement (Lonigo, Legnago, San Bonifacio, and Noventa Vicentina).

All the above analyses have been also stratified according to gender and an interaction term between gender and ln-PFAS was also added to the main models.

Since PFAS are predominantly excreted by the kidney through glomerular filtration and impaired kidney function is associated with raised BP [23], to assess for possible confounding a sensitivity analysis was conducted adjusting all models for estimated glomerular filtration rate (eGFR with cut-off $<90 \mathrm{~mL} / \mathrm{min}$ ) calculated according to the CKD-EPI equation [26].

Finally, we analysed SBP and DBP associations with PFAS excluding subjects with raised BP $(\mathrm{SBP} \geq 140$ $\mathrm{mmHg}$ or DBP $\geq 90 \mathrm{mmHg}$ ).

The procedures of the health surveillance program changed over time: until 31 December 2017, blood sampling, and interview and BP measurement were carried out in the same session for each participant, whilst thereafter they were performed in two different sessions roughly 1 month apart in order to be able to provide blood test results on the day the participant came for the interview and the BP measurement. To explore whether this organizational change may have affected the PFAS/BP associations, analyses were also restricted to the subgroup of 10,656 individuals recruited after 31 December 2017.

The level of statistical significance was set at 0.05 . The statistical software STATA/SE version 13.0 (Stata Corp LP, College Station, TX, USA) and R (R Development Core Team 2010, R Foundation for Statistical Computing, Vienna, Austria. ISBN 3-900,051-07-0, URL: http:// www.R-project.org/) was used for statistical analyses.

\section{Results}

Table 1 provides descriptive results on PFAS, outcome variables and selected covariates of the study population. 7667 (49\%) males and 8119 (51\%) females were included in the analyses with a mean age of 30 years (SD 5.9).
Further characteristics of participants according to gender are reported in Supplementary Table 1, Additional File 2.

Among the four PFAS, PFOA was detected at the highest level (median $35.8 \mathrm{ng} / \mathrm{mL}$ ), followed by PFOS (median $3.7 \mathrm{ng} / \mathrm{mL}$ ), PFHxS (median $3.6 \mathrm{ng} / \mathrm{mL}$ ), and PFNA (median $0.35 \mathrm{ng} / \mathrm{mL}$ ). The highest serum levels of all PFAS were found in males compared to females. Moderate to strong correlations were observed among the measured PFAS. The most highly correlated compounds were PFHxS with PFOA $(\rho=0.91)$; while the least correlated were PFNA with PFOA and PFHxS (both $\rho=0.40$ ). The Spearman correlation coefficients of PFOS with PFOA and PFHxS were 0.63 and 0.68 , respectively.

Hypertension was detected in 1971 (12.5\%) of the recruited subjects (19\% in males and 6\% in females). Significant differences in demographic and lifestyle characteristics according to hypertensive status were observed among participants (See Supplementary Table 2, Additional File 3). In particular, hypertensive subjects were older, had a lower educational level, and a higher BMI. Moreover, significant differences in the prevalence of hypertension were found between centers in charge for blood pressure measurement.

Table 2 depicts the results of the fitted models assessing the association between each PFAS, considered as ln-linear predictors or categorical predictors (based on quartiles), and SBP and DBP, after adjustment for confounding factors. As shown in Table 2, each $\ln$-increase in PFOA was associated with an increase in SBP of 0.37 $\mathrm{mmHg}$ (95\%CI $0.19-0.54), 0.57 \mathrm{mmHg}$ (95\%CI $0.24-$ 0.90) for PFOS, $0.37 \mathrm{mmHg}$ (95\%CI $0.15-0.58$ ) for PFHxS, and $0.99 \mathrm{mmHg}$ (95\%CI $0.47-1.51)$ for PFNA. Similarly, each $\ln$-increase in PFOA was associated with an increase in DBP of $0.34 \mathrm{mmHg}$ (95\%CI $0.21-0.47$ ), $0.44 \mathrm{mmHg}$ (95\%CI $0.20-0.68)$ for PFOS, $0.33 \mathrm{mmHg}$ (95\% CI $0.17-0.48)$ for $\mathrm{PFHxS}$, and $0.62 \mathrm{mmHg}$ (95\%CI 0.24-1.00) for PFNA. Subjects in the highest PFOA quartile had respectively $1.07 \mathrm{mmHg}$ (95\%CI $0.46-1.68)$ and $0.97 \mathrm{mmHg}(95 \% \mathrm{CI} 0.53-1.42)$ higher SBP and DBP than those in the lowest quartile. Slightly lower increments were seen for the other PFAS. The predicted increase in SBP and DBP were $1.54 \mathrm{mmHg}(95 \% \mathrm{CI} 0.61-$ 2.47), $1.60 \mathrm{mmHg}$ (95\% CI $0.92-2.27$ ) from lowest to highest decile of PFOA and $1.25 \mathrm{mmHg}(95 \% \mathrm{CI} 0.36-$ 2.14), $1.02 \mathrm{mmHg}$ (95\%CI $0.37-1.67$ ) from lowest to highest decile of PFOS (See Supplementary Figure 2, Additional File 4).

Furthermore, the adjusted concentration-response curves between SBP, DBP and PFAS in the total studied population are shown in Fig. 1 and the results stratified by gender are presented in the Supplementary Figure 3, Additional File 5. These analyses revealed highly 
Table 1 Characteristics of the study population $(n=15,786)$, stratified by gender

\begin{tabular}{|c|c|c|c|c|c|c|c|c|c|}
\hline \multirow[t]{2}{*}{ Variables } & \multicolumn{3}{|c|}{ Total $(n=15,786)$} & \multicolumn{3}{|c|}{ Males $(n=7667)$} & \multicolumn{3}{|c|}{ Females $(n=8119)$} \\
\hline & mean (SD) & $\min -\max$ & $\begin{array}{l}\text { Median } \\
\text { (Q1-Q3) }\end{array}$ & mean (SD) & $\min -\max$ & $\begin{array}{l}\text { Median } \\
\text { (Q1-Q3) }\end{array}$ & mean (SD) & $\min -\max$ & $\begin{array}{l}\text { Median } \\
\text { (Q1-Q3) }\end{array}$ \\
\hline \multicolumn{10}{|l|}{ PFAS } \\
\hline PFOA (ng/mL) & $59.69(72.26)$ & $0.35-1400$ & $35.8(13.7-78.9)$ & $83.84(87.51)$ & $0.35-1400$ & $58.3(25.1-114.7)$ & 36.89 (43.03) & $0.35-671$ & $22.6(8.8-49.4)$ \\
\hline PFOS (ng/mL) & $4.63(4.02)$ & $0.35-142$ & $3.7(2.5-5.6)$ & $5.72(4.43)$ & $0.35-142$ & $4.8(3.3-6.9)$ & $3.59(3.27)$ & $0.35-124$ & $3(2-4.4)$ \\
\hline PFHxS (ng/mL) & $5.97(6.82)$ & $0.35-127$ & $3.6(1.6-7.8)$ & $8.86(8.29)$ & $0.35-127$ & $6.5(3-12)$ & $3.24(3.19)$ & $0.35-41.3$ & $2.2(1.1-4.3)$ \\
\hline PFNA (ng/mL) & $0.53(0.42)$ & $0.35-39.7$ & $0.35(0.35-0.6)$ & $0.59(0.52)$ & $0.35-39.7$ & $0.5(0.35-0.7)$ & $0.48(0.28)$ & $0.35-8.8$ & $0.35(0.35-0.5)$ \\
\hline \multicolumn{10}{|l|}{ Covariates } \\
\hline Age (years) & $30.04(5.86)$ & $20-39$ & $30(25-35)$ & 29.88 (5.88) & $20-39$ & $30(25-35)$ & $30.2(5.84)$ & $20-39$ & $31(25-35)$ \\
\hline BMI $\left(\mathrm{kg} / \mathrm{m}^{2}\right)$ & $23.91(4.3)$ & $13.58-50.47$ & $23.27(20.9-26.04)$ & $24.75(3.82)$ & $13.58-49.01$ & $24.22(22.23-26.59)$ & $23.11(4.56)$ & $14.32-50.47$ & $22.04(19.92-25.08)$ \\
\hline $\begin{array}{l}\text { Time-lag } \\
\text { (months) }\end{array}$ & $14.77(5.45)$ & $3-28$ & $14(10-19)$ & $14.7(5.51)$ & $5-28$ & $14(10-19)$ & $14.84(5.39)$ & $3-28$ & $14(10-19)$ \\
\hline Gender & n (\%) & & & & & & & & \\
\hline Male & 7667 (48.57) & & & & & & & & \\
\hline Female & 8119 (51.43) & & & & & & & & \\
\hline Education & n (\%) & & & n (\%) & & & n (\%) & & \\
\hline $\begin{array}{l}\text { Elementary/ } \\
\text { Middle school }\end{array}$ & 2409 (15.26) & & & $1346(17.56)$ & & & $1063(13.09)$ & & \\
\hline Highschool & $9445(59.83)$ & & & $4847(63.22)$ & & & 4598 (56.63) & & \\
\hline University & $3932(24.91)$ & & & $1474(19.23)$ & & & $2458(30.27)$ & & \\
\hline Physical activity & n (\%) & & & n (\%) & & & n (\%) & & \\
\hline Light & 10,638 (67.39) & & & 4719 (61.55) & & & 5919 (72.9) & & \\
\hline Moderate & $2394(15.17)$ & & & $1152(15.03)$ & & & $1242(15.3)$ & & \\
\hline Heavy & 2754 (17.45) & & & $1796(23.43)$ & & & $958(11.8)$ & & \\
\hline Smoke & n (\%) & & & n (\%) & & & n (\%) & & \\
\hline NO & $9230(58.47)$ & & & 3895 (50.8) & & & $5335(65.71)$ & & \\
\hline YES & 4355 (27.59) & & & 2575 (33.59) & & & 1780 (21.92) & & \\
\hline Ex & $2201(13.94)$ & & & $1197(15.61)$ & & & 1004 (12.37) & & \\
\hline Country of birth & n (\%) & & & n (\%) & & & n (\%) & & \\
\hline HDC & $14,297(90.57)$ & & & 7136 (93.07) & & & 7161 (88.2) & & \\
\hline HMPC & $1489(9.43)$ & & & $531(6.93)$ & & & $958(11.8)$ & & \\
\hline \multicolumn{10}{|l|}{ Outcomes } \\
\hline & n (\%) & & & n (\%) & & & n (\%) & & \\
\hline \multirow[t]{2}{*}{ Hypertension } & 1971 (12.49) & & & $1449(18.9)$ & & & $522(6.43)$ & & \\
\hline & \multicolumn{3}{|c|}{ Total $(n=15,380)$} & \multicolumn{3}{|c|}{ Males $(n=7428)$} & \multicolumn{3}{|c|}{ Females $(n=7952)$} \\
\hline Blood pressure & mean $(S D)$ & $\min -\max$ & Median (Q1-Q3) & mean $(S D)$ & $\min -\max$ & Median (Q1-Q3) & mean (SD) & $\min -\max$ & Median (Q1-Q3) \\
\hline $\begin{array}{l}\text { Systolic BP } \\
(\mathrm{mmHg})\end{array}$ & 119.77 (14.23) & $70-180$ & $120(110-130)$ & $125.08(13.51)$ & $70-180$ & $125(118-134)$ & $114.8(13.04)$ & $70-180$ & $115(105-122)$ \\
\hline $\begin{array}{l}\text { Diastolic BP } \\
(\mathrm{mmHg})\end{array}$ & $74.36(9.74)$ & $25-119$ & $75(70-80)$ & $76.36(9.58)$ & $25-119$ & $79(70-81)$ & $72.51(9.51)$ & $40-112$ & $72(65-80)$ \\
\hline
\end{tabular}

significant associations between both SBP and DBP and all investigated PFAS with approximately linear relationships between the natural logarithm of the four PFAS and these outcomes, thus showing a linear-log relationship with the back transformed PFAS values (See Supplementary Table 3, Additional File 6).

Gender significantly modified the association between all PFAS and SBP, with significant associations seen almost exclusively among men; on the contrary, women showed stronger associations with DBP (although with no significant $p$-value for interaction). Adjusting for eGFR did not change the results of the observed associations between PFAS and SBP or DBP (See Supplementary Table 4, Additional File 7). The analyses on the restricted population recruited after 31 December 2017 also showed similar results (See Supplementary Table 5, 
Table 2 Association between PFAS (In ng/mL) and systolic and diastolic blood pressure (mmHg from GAM models ( $n=15^{\prime} 380$ ), adjusted by several covariates: $\beta$ coefficients and 95\% Confidence Intervals (Cl)

\begin{tabular}{|c|c|c|c|c|c|c|c|c|c|c|c|c|}
\hline \multirow[t]{3}{*}{ PFAS } & \multicolumn{6}{|c|}{ Systolic Blood Pressure } & \multicolumn{6}{|c|}{ Diastolic Blood Pressure } \\
\hline & \multicolumn{2}{|l|}{ Total } & \multicolumn{2}{|l|}{ Males } & \multicolumn{2}{|c|}{ Females } & \multicolumn{2}{|l|}{ Total } & \multicolumn{2}{|l|}{ Males } & \multicolumn{2}{|c|}{ Females } \\
\hline & $\bar{\beta}$ & $\mathrm{Cl} 95 \%$ & $\bar{\beta}$ & $\mathrm{Cl} 95 \%$ & $\bar{\beta}$ & $\mathrm{Cl} 95 \%$ & $\beta$ & $\mathrm{Cl} 95 \%$ & $\beta$ & $\mathrm{Cl} 95 \%$ & $\bar{\beta}$ & $\mathrm{Cl} 95 \%$ \\
\hline In_PFOA & 0.37 & $0.19-0.54$ & 0.46 & $0.19-0.73$ & 0.31 & $0.08-0.55$ & 0.34 & $0.21-0.47$ & 0.23 & $0.04-0.42$ & 0.39 & $0.21-0.57$ \\
\hline IQ & 113.81 & & 122.08 & & 113.09 & & 73.27 & & 77.4 & & 72.38 & \\
\hline$\| Q$ & 0.26 & $-0.29-0.81$ & 0.12 & $-0.87-1.1$ & 0.46 & $-0.2-1.13$ & 0.24 & $-0.16-0.64$ & -0.31 & $-1-0.38$ & 0.44 & $-0.06-0.94$ \\
\hline III Q & 0.74 & $0.16-1.31$ & 0.8 & $-0.15-1.75$ & 0.8 & $0.06-1.53$ & 0.78 & $0.36-1.2$ & 0.4 & $-0.27-1.07$ & 0.74 & $0.19-1.3$ \\
\hline IV Q & 1.07 & $0.46-1.68$ & 1.25 & $0.32-2.18$ & 0.71 & $-0.2-1.62$ & 0.97 & $0.53-1.42$ & 0.49 & $-0.17-1.14$ & 1.15 & $0.46-1.83$ \\
\hline In_PFOS & 0.57 & $0.24-0.9$ & 0.98 & $0.47-1.48$ & 0.32 & $-0.13-0.77$ & 0.44 & $0.2-0.68$ & 0.29 & $-0.07-0.64$ & 0.51 & $0.17-0.84$ \\
\hline IQ & 114.15 & & 122.21 & & 114.34 & & 73.51 & & 77.51 & & 72.67 & \\
\hline$\| \mathrm{Q}$ & -0.01 & $-0.56-0.53$ & 0.46 & $-0.55-1.47$ & -0.07 & $-0.72-0.58$ & 0.32 & $-0.08-0.72$ & 0.3 & $-0.41-1.01$ & 0.24 & $-0.24-0.73$ \\
\hline III Q & 0.27 & $-0.29-0.84$ & 0.79 & $-0.18-1.76$ & 0.26 & $-0.47-0.98$ & 0.3 & $-0.12-0.71$ & 0.12 & $-0.57-0.8$ & 0.26 & $-0.29-0.8$ \\
\hline IV Q & 0.6 & $0-1.21$ & 1.13 & $0.16-2.09$ & 0.42 & $-0.45-1.29$ & 0.57 & $0.13-1.02$ & 0.19 & $-0.49-0.87$ & 1.00 & $0.34-1.66$ \\
\hline In_PFHXS & 0.37 & $0.15-0.58$ & 0.61 & $0.3-0.93$ & 0.17 & $-0.13-0.47$ & 0.33 & $0.17-0.48$ & 0.29 & $0.07-0.51$ & 0.26 & $0.04-0.49$ \\
\hline IQ & 113.983 & & 121.71 & & 113.4 & & 73.37 & & 77.03 & & 72.65 & \\
\hline$\| \mathrm{Q}$ & -0.01 & $-0.56-0.54$ & 0.63 & $-0.43-1.69$ & -0.14 & $-0.78-0.51$ & 0.2 & $-0.2-0.6$ & 0.49 & $-0.25-1.24$ & 0.04 & $-0.44-0.52$ \\
\hline III Q & 0.61 & $0.04-1.18$ & 1.16 & $0.14-2.17$ & 0.57 & $-0.14-1.28$ & 0.7 & $0.28-1.11$ & 0.74 & $0.03-1.46$ & 0.57 & $0.03-1.11$ \\
\hline IV Q & 0.93 & $0.3-1.57$ & 1.54 & $0.55-2.53$ & 0.43 & $-0.61-1.46$ & 0.78 & $0.32-1.25$ & 0.75 & $0.05-1.45$ & 0.75 & $-0.03-1.53$ \\
\hline In_PFNA & 0.99 & $0.47-1.51$ & 1.46 & $0.72-2.19$ & 0.54 & $-0.21-1.28$ & 0.62 & $0.24-1$ & 0.36 & $-0.16-0.88$ & 0.81 & $0.25-1.37$ \\
\hline
\end{tabular}

Additional File 8) as the one limited to those subjects with SBP and DBP within normal ranges (See Supplementary Table 6, Additional File 9).

Table 3 presents results of multivariable logistic models for the association between PFAS levels as lnlinear predictors or categorical predictors (based on quartiles) and hypertension prevalence. One unit increase in each ln-PFAS was positively associated with an increased odds of hypertension after controlling for potential confounders: PFOA OR $=1.06$ (95\%CI 1.01-1.12), PFOS OR $=1.12(95 \%$ CI 1.02-1.22), $\mathrm{PFHxS}$ OR $=1.08$ (95\%CI 1.02-1.15), PFNA OR $=1.10$ (95\%CI 0.96-1.26). When stratified by gender, positive associations were detected only in men, with the strongest effect seen for PFNA OR $=1.19 \quad(95 \% \mathrm{CI} \quad 1.02-1.40) \quad(p$-value interaction $=0.048)$.

\section{Discussion}

In the present cross-sectional study consisting of more than 15,000 young adults from a highly exposed community, serum PFAS concentrations displayed a positive association with blood pressure levels and were also associated with a raised prevalence of hypertension in men. The magnitude of the association with blood pressure was relatively small and for/regarding systolic blood pressure, was significantly modified by gender, with associations seen almost only for men.

To the best of our knowledge, this is the first study that formally investigated the shape of the association between serum PFAS and blood pressure in a large population of highly exposed community residents (more than 15,000 participants) consisting of a homogeneous age group of young adults from 20 to 39 years old. Literature on the association between PFAS exposure and blood pressure and/or hypertension (defined as selfreported diagnosis or treatment or elevated measured blood pressure) is relatively limited, with contradictory findings. Some large cross-sectional studies were conducted on representative samples of the US general population with background exposure using data from the NHANES survey [11, 12, 14, 18, 19]. Among 2934 adults, Min et al. showed a significant increase in SBP, but not DBP, associated with increasing PFOA concentrations [12]. Moreover, the Odds Ratio of hypertension was 2.62 when comparing the 80th and 20th percentiles of the PFOA distribution. In contrast, $\mathrm{He}$ et al. $(n=$ 7543) reported no significant association with blood pressure, but blood pressure was not the primary outcome of this study [19]. In a recent cross-sectional study on 6967 adults from NHANES, Liao et al. found a positive log-linear association of serum PFOA, PFOS, and PFHxS with SBP and a non-linear J-shaped association of serum PFOS and PFNA with DBP; moreover, the study showed a non-linear J-shaped association of serum PFOA and PFNA with the odds of hypertension [14]. The other two studies focused on children and adolescents with background exposure to PFAS $[11,18]$, yielding conflicting results: while Geiger et al. $(n=1655)$ 


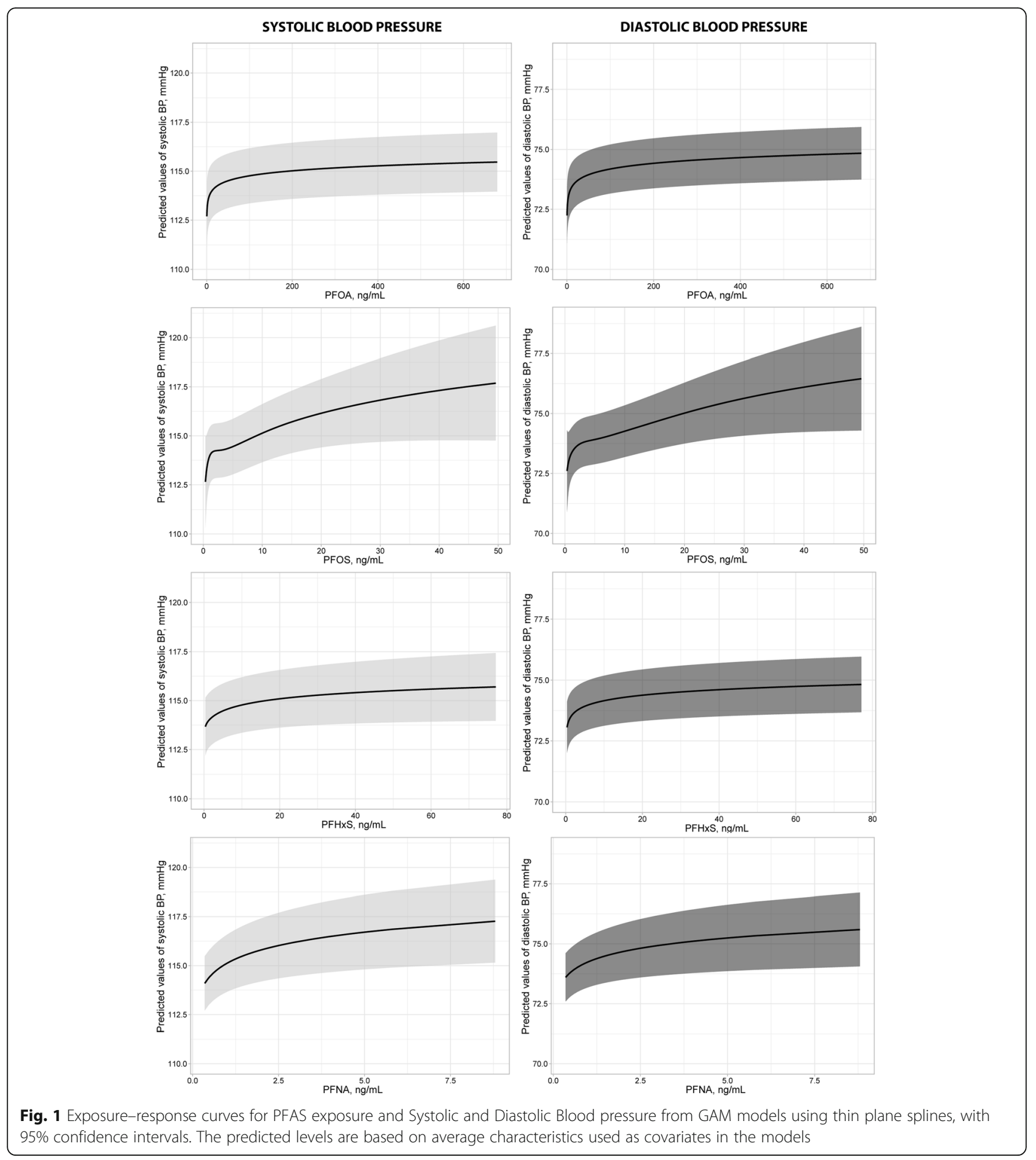

found no evidence of an association between serum PFAS and blood pressure or hypertension [18], Ma et al. $(n=2251)$ found a positive significant association between PFOS and DBP among males [11]. Other crosssectional studies with smaller sample size (range $n=48-$ $187)$ reported positive $[10,13]$, null $[15,17]$ or negative associations [16]. A study on highly exposed Chinese adults (China C8 Health Project, $n=1612$ ) investigated the association between blood pressure and several PFAS congeners, including linear and branched isomers [9]. Significant positive associations emerged for logtransformed PFOS, PFOA, PFNA, PFBA, and PFPeA, but not PFHxS. As for PFOA, the association was attributable only to branched isomers. Moreover, a strong 
Table 3 Association between PFAS and risk of hypertension from GAM models adjusted by several covariates, using PFAS quartiles ( $\mathrm{n}=15,786)$ : odds ratios (OR) and 95\% Confidence Intervals (Cl)

\begin{tabular}{|c|c|c|c|c|c|c|c|c|c|}
\hline \multirow[t]{2}{*}{ PFAS } & \multicolumn{3}{|l|}{ Total } & \multicolumn{3}{|c|}{ Males } & \multicolumn{3}{|c|}{ Females } \\
\hline & $\overline{\mathrm{OR}}$ & CI Lower & $\overline{\mathrm{Cl} \text { Upper }}$ & $\overline{\mathrm{OR}}$ & CI Lower & $\overline{\mathrm{Cl} \text { Upper }}$ & $\overline{\mathrm{OR}}$ & Cl Lower & CI Upper \\
\hline In_PFOA & 1.06 & 1.01 & 1.12 & 1.08 & 1.02 & 1.15 & 1.06 & 0.97 & 1.15 \\
\hline IQ & 1 & & & 1 & & & 1 & & \\
\hline$\| \mathrm{Q}$ & 1.00 & 0.85 & 1.16 & 1.00 & 0.81 & 1.24 & 1.05 & 0.84 & 1.32 \\
\hline\|\| $\mathrm{Q}$ & 1.02 & 0.87 & 1.20 & 1.11 & 0.90 & 1.36 & 0.93 & 0.71 & 1.23 \\
\hline IV Q & 1.16 & 0.99 & 1.37 & 1.21 & 0.99 & 1.48 & 1.17 & 0.84 & 1.62 \\
\hline In_PFOS & 1.12 & 1.02 & 1.22 & 1.17 & 1.05 & 1.31 & 1.06 & 0.91 & 1.24 \\
\hline IQ & 1 & & & 1 & & & 1 & & \\
\hline$\| \mathrm{Q}$ & 0.99 & 0.85 & 1.16 & 1.10 & 0.88 & 1.37 & 0.97 & 0.77 & 1.22 \\
\hline III Q & 1.06 & 0.91 & 1.24 & 1.26 & 1.02 & 1.55 & 0.85 & 0.65 & 1.12 \\
\hline IV Q & 1.12 & 0.95 & 1.32 & 1.28 & 1.03 & 1.58 & 1.02 & 0.74 & 1.41 \\
\hline In_PFHxS & 1.08 & 1.02 & 1.15 & 1.11 & 1.03 & 1.19 & 1.08 & 0.97 & 1.20 \\
\hline IQ & 1 & & & 1 & & & 1 & & \\
\hline$\| \mathrm{Q}$ & 1.01 & 0.86 & 1.19 & 1.08 & 0.85 & 1.37 & 1.01 & 0.80 & 1.26 \\
\hline\|\| $\mathrm{Q}$ & 1.08 & 0.92 & 1.27 & 1.19 & 0.95 & 1.50 & 1.00 & 0.77 & 1.30 \\
\hline IV Q & 1.19 & 1.00 & 1.41 & 1.27 & 1.02 & 1.59 & 1.28 & 0.90 & 1.83 \\
\hline In_PFNA & 1.10 & 0.96 & 1.26 & 1.19 & 1.02 & 1.40 & 0.94 & 0.71 & 1.25 \\
\hline
\end{tabular}

gender difference was found, with more significant associations among females [9].

To our knowledge, only two studies assessed the association between PFAS exposure and blood pressure or hypertension with a longitudinal design [21, 22]. In the C8 Health Project, a large study involving a highly exposed community of the Mid-Ohio Valley, USA, there was no clear evidence of an association between PFOA exposure and self-reported incident hypertension, although small statistically significant associations emerged in certain subgroups (females aged 20-39 years and males aged $40-59$ years) [21]. Lin and colleagues explored the issue using data from a randomized controlled trial involving 957 pre-diabetic patients that underwent either a lifestyle intervention or standard care. At baseline, they found a positive cross-sectional association between PFOA serum levels and SBP and a significantly increased prevalence of hypertension with higher serum PFOA among males. In the prospective analyses, however, the associations with both blood pressure trajectories and the risk of developing hypertension were null, except for an inverse association between PFOS and SBP in the intervention arm [22].

As outlined above, the available literature shows several inconsistencies: the direction, statistical significance, and magnitude of associations, as well as the gender effect modification, were different among studies. This may be attributable to the heterogeneous characteristics of the investigated populations, in terms of age, social context, and levels of exposure. Moreover, reported effect sizes are poorly comparable with each other. The two studies more comparable with the present one in terms of design and population size were conducted on the general US population with exposure levels quite different from ours $[9,12]$. Min et al. reported median serum PFOA of $4 \mathrm{mcg} / \mathrm{L}$, and Bao et al. reported median serum PFOA of $6.19 \mathrm{ng} / \mathrm{ml}$ and median serum PFOS of $24.22 \mathrm{ng} / \mathrm{ml}$. Both studies found associations of greater magnitude than ours. Min and colleagues reported linear regression coefficients between log-transformed serum PFOA and SBP of 2.48 and 0.75 for DBP. In the study by Bao et al., for each ln-unit increase of serum PFOA, PFOS, and PFNA, SBP increased by $1.69,4.84$, and 3.01 $\mathrm{mmHg}$, and DBP increased by 2.18, 2.70, and 2.48 $\mathrm{mmHg}$, respectively.

Although the magnitude of the observed effect was relatively small, if confirmed it would be of public health relevance since the greatest magnitude of effect was observed at lower serum PFAS concentrations corresponding to background exposure and it is widely recognized that even small increases in blood pressure levels imply a raised risk of adverse outcomes such as CVD and target organ damage [27]. It has been estimated that a 1 mmHg-reduction of SBP at the population level may be associated to a decrease in the incidence rate for 100,000 person-years of 9 coronary heart disease events, 4.8 stroke events, and 13.3 heart failure events [28]. Of note, the detrimental long-term effects of blood pressure elevation have been demonstrated also in young adults [29]. Nevertheless, only few studies have investigated the 
association between PFAS and CVD so far, with inconsistent findings [21, 30-33]. In the C8 Health Study, cumulative serum PFOA was associated with a significantly increased risk of stroke with a non-monotonic doseresponse relationship [33]. The association with the risk of coronary artery disease was less clear since it was present only in males of certain age subgroups [21].

The biological mechanisms underlying the association between PFAS exposure and elevated blood pressure have still to be fully elucidated. Potential biological pathways may include oxidative stress leading to impaired vasodilation [34-37]. Oxidative stress is proposed to play a critical role in the pathogenesis of hypertension [38, 39]. Moreover, intra-uterine exposure has been shown to reduce the number of nephrons at birth [40], which may be associated with over-activation of existing nephrons, pressure natriuresis, and blood pressure elevation. This hypothesis would be compatible with our findings since our study population was composed of young adults that may have been exposed to PFAS since conception. Furthermore, PFAS might interfere with the signaling pathways of the thyroid hormones [7] which have an important role in energy metabolism and blood pressure regulation. Therefore, PFAS may influence blood pressure with a combination of various physiological processes such as alteration of the levels of thyroid hormones or level of total cholesterol as indirect mechanism of actions [41]. Furthermore, PFOS as potential endocrine disrupting chemical was also related to the transcriptional induction of the gene cyp11b2 (encoding CYP11B2, aldosterone synthetase). Aldosterone is involved in blood pressure regulation by increasing the reuptake of ions and water in the kidneys [42].

The observed gender differences were inconsistent between studies $[9,11]$ and require further investigations. The fact that in our study the association with SBP and hypertension was present mainly in men may reflect the lower serum PFAS levels and lower prevalence of hypertension among women. Women had a higher proportion of PFAS levels below the quantification limit, which makes more difficult to know the real PFAS levels in this subgroup. The half-life difference in males and females [43] may be one of the underlying factors affecting the difference in the results according to gender. Those differences may also be related to the effect of sex hormones on blood pressure regulation in a young adult population like the one of the present study. In one hand, estradiol has been shown to promote peripheral vasodilation with ensuing lower blood pressure, and on the other hand menopause is associated to an increase of blood pressure levels compared to the reproductive age [44]. Some in vitro studies indicate that PFAS may interfere with the signaling of human sex hormones [45-47], which may also contribute to the observed gender differences of the association between PFAS and blood pressure.
Strengths of this study include the large population and the availability of an accurate measure of the internal dose of PFAS and of information on several anthropometric, lifestyle, and clinical variables, that allowed adjustment for many possible confounders. The high dispersion of serum PFOA concentrations in our population allowed to model concentration-response curves over a wide range of internal doses, covering both background and high exposure levels. Moreover, we systematically evaluated gender-specific associations. The main limitation is the cross-sectional design that precludes evaluation of the temporal relationship between exposure and outcome. A limitation in looking at individual PFAS is that they are highly correlated. Especially for the compounds with lower serum levels, such as PFNA in our study, the association may reflect associations driven by the correlated PFAS at higher concentration. The high correlation also prevents multipollutant models to assess their mutual adjustment. Also, we were unable to investigate the role of different PFAS isomers since only total PFAS serum concentrations were available. Another important limitation is that in most subjects we relied on a single blood pressure measurement. Blood pressure is subject to high intra-individual variation, therefore a single measurement may be poorly representative of an individual's mean blood pressure [23]. Moreover, results of blood pressure measurements are both device- and operator-dependent [48]. Indeed, we observed a significant difference of hypertension prevalence between centers in charge for blood pressure measurement. Random intra-individual variation would cause a non-differential misclassification of outcome and therefore would bias associations towards the null. By contrast, device- and operator-dependence may have introduced a systematic misclassification; however, to control for this we adjusted all analyses by center in charge for blood pressure measurement. The change in the procedures occurred after December 2017 may have provoked a differential misclassification of outcome in the case participants were informed of their serum PFAS levels prior to blood pressure measurement, possibly resulting in higher blood pressure among subjects with higher PFAS levels due to alarm reaction. To address this issue, we restricted analyses to participants recruited after 31 December 2017, but we found no evidence of such a systematic error since results were very similar to the overall analyses.

\section{Conclusions}

In conclusion, our findings suggest that serum PFAS concentrations are associated with raised blood pressure levels with the greatest increases seen at the lower internal dose range. These are cross-sectional associations and should be interpreted with caution and the average 
change in blood pressure is quite modest. However, PFAS exposure is widespread and so if the association is causal, it would imply a significant population health impact. Such findings should be confirmed by further studies with a longitudinal design and more refined methods to measure blood pressure levels. Moreover, longitudinal studies are warranted to assess whether PFAS exposure is a risk factor for the development of cardiovascular events.

\section{Supplementary information}

Supplementary information accompanies this paper at https://doi.org/10. 1186/s12940-020-00656-0.

\section{Additional file 1: Figure 1. Flowchart of the study population.}

Additional file 2: Table 1. Descriptive statistics of diet and center where Blood Pressure has been measured.

Additional file 3: Table 2. Descriptive statistics in subjects with or without hypertension, stratified by gender.

Additional file 4: Figure 2. Exposure-response curves for PFAS exposure and Systolic and Diastolic Blood pressure from GAM models using thin plane splines, with 95\% confidence intervals, stratified by gender. The predicted levels are based on average characteristics used as covariates in the models.

Additional file 5: Figure 3. Predicted values of Systolic and Diastolic Blood Pressure by deciles of PFAS distribution.

Additional file 6: Table 3. GAM model with using PFAS thin plate spline smooth terms: EDF and $p$-values.

Additional file 7: Table 4. GAM models adjusted by eGFR (cut-off 90 $\mathrm{ml} / \mathrm{min}$ )

Additional file 8: Table 5. GAM models on a restricted population recruited after December $2017(n=10,656)$

Additional file 9: Table 6. GAM models on a restricted population, excluding subclinical hypertensive $(n=13,815)$.

\section{Abbreviations}

PFAS: Perfluoroalkyl substances; SBP: Systolic blood pressure; DBP: Diastolic blood pressure; PFOS: Perfluorooctanesulfonate; PFOA: Perfluorooctanoic acid; PFHXS: Perfluorohexanesulfonic acid; PFNA: Perfluorononanoic acid; PFHpA: Perfluoroheptanoic acid; PFBS: Perfluorobutanesulfonic acid; PFHxA: Perfluorohexanoic acid; PFBA: Perfluorobutanoic acid; PFPeA: Perfluoropentanoic acid; PFDeA: Perfluorodecanoic acid; PFUnA: Perfluoroundecanoic acid; PFDoA: Perfluorododecanoic acid; LOD: Limit of detection; LOQ: Limit of quantification; BP: Blood pressure; BMI: Body mass index; DAG: Directed acyclic graph; In-PFAS: Natural log (In) transformed PFAS; GAMs: Generalized additive models; eGFR: Estimated glomerular filtration rate

\section{Acknowledgements}

The authors gratefully acknowledge the contributions of Filippo Da Re (Regional Directorate of Prevention, Food Safety, Veterinary Public Health Regione del Veneto), Elena Narne (Screening and Health Impact Assessment Unit - Azienda Zero), Rinaldo Zolin (Local Health Unit 8 Berica), Annamaria Bettega (Local Health Unit 8 Berica), Lorena Zambelli (Local Health Unit 9 Scaligera), Katia Grego (Local Health Unit 9 Scaligera), Daniele Bottigliengo, lleana Baldi, Dario Gregori (University of Padova).

\section{Authors' contributions}

GP contributed to the conception and design of the Veneto surveillance plan, interpreted the results and wrote the original draft. GB analyzed and interpreted the data. MZJ, MG, AF interpreted the results, reviewed drafts of the manuscript and provided valuable comments for the manuscript. FR is responsible of the Veneto surveillance plan, reviewed drafts and provided valuable comments for the manuscript. FD is responsible for the chemical analysis of PFAS in the study, reviewed the drafts and provided valuable comments for the manuscript. TF supervised the methodology, interpreted the results, reviewed drafts of the manuscript and provided valuable comments for the manuscript. CC is the principal investigator, led the conceptual and methodological design of the study, interpreted the results and wrote the original draft. The author(s) read and approved the final manuscript.

\section{Funding}

RIS/REGIONE VENETO (IT) supported this research with a grant to Cristina Canova. Award Number: CANO_ALFREVE18_01 (CONVENZIONE CORIS PER REALIZZAZIONE PROGETTI DI RICERCA INNOVATIVI SUI PFAS 2017-18). The funder had no role in study design, data collection and analysis, decision to publish, or preparation of the manuscript.

\section{Availability of data and materials}

Data contains sensitive personal information and cannot be made publicly available. Any data inquiries are referred to the corresponding author (cristina.canova@unipd.it).

Ethics approval and consent to participate

All participants gave informed consent to take part in the health surveillance program.

\section{Consent for publication}

Not applicable.

\section{Competing interests}

The authors have declared that no competing interests exist.

\section{Author details}

${ }^{1}$ Screening and Health Impact Assessment Unit, Azienda Zero-Veneto Region, Padova, Italy. ${ }^{2}$ Unit of Biostatistics, Epidemiology and Public Health, Department of Cardio-Thoraco-Vascular Sciences and Public Health, Via Loredan 18, 35131 Padova, Italy. ${ }^{3}$ Regional Center for Biomarkers, Department of Clinical Pathology, Azienda ULSS 3 Serenissima, Venice, Italy. ${ }^{4}$ Laboratory Department-Regional Agency for Environmental Prevention and Protection-Veneto Region, Venice, Italy. ${ }^{5}$ Directorate of Prevention, Food Safety, and Veterinary Public Health-Veneto Region, Venice, Italy. '́London School of Hygiene and Tropical Medicine, London, UK.

Received: 29 July 2020 Accepted: 11 September 2020 Published online: 21 September 2020

\section{References}

1. OECD, The Organisation for Economic Co-operation and Development Toward a New Comprehensive Global Database of Per- and Polyfluoroalkyl Substances (PFASs): Summary Report on Updating the OECD 2007 List of per and Polyfluoroalkyl Substances (PFASs)'. Retrieved 13 June 2019. 2018.

2. Poothong S, Papadopoulou E, Padilla-Sánchez JA, Thomsen C, Haug LS. Multiple pathways of human exposure to poly- and perfluoroalkyl substances (PFASs): from external exposure to human blood. Environ Int. 2020;134:105244.

3. Frisbee SJ, Brooks AP, Maher A, Flensborg P, Arnold S, Fletcher T, et al. The C8 health project: design, methods, and participants. Environ Health Perspect. 2009;117:1873-82.

4. Hölzer J, Midasch $O$, Rauchfuss $K$, Kraft M, Reupert R, Angerer J, et al. Biomonitoring of perfluorinated compounds in children and adults exposed to perfluorooctanoate-contaminated drinking water. Environ Health Perspect. 2008;116:651-7.

5. Li Y, Barregard L, Xu Y, Scott K, Pineda D, Lindh CH, et al. Associations between perfluoroalkyl substances and serum lipids in a Swedish adult population with contaminated drinking water. Environ Health. 2020;19:33.

6. Pitter G, Da Re F, Canova C, Barbieri G, Zare Jeddi M, Daprà F, et al. Serum levels of Perfluoroalkyl substances (PFAS) in adolescents and young adults exposed to contaminated drinking water in the Veneto region, Italy: A Cross-Sectional Study Based on a Health Surveillance Program. Environ Health Perspect. 2020;128:027007.

7. EFSA Panel on contaminants in the food chain (CONTAM). Risk to human health related to the presence of perfluorooctane sulfonic acid and perfluorooctanoic acid in food 2018. 
8. Zhou B, Bentham J, Di Cesare M, Bixby H, Danaei G, Cowan MJ, et al. Worldwide trends in blood pressure from 1975 to 2015: a pooled analysis of 1479 population-based measurement studies with 19.1 million participants. Lancet. 2017;389:37-55.

9. Bao W-W, Qian Z, Geiger SD, Liu E, Liu Y, Wang S-Q, et al. Gender-specific associations between serum isomers of perfluoroalkyl substances and blood pressure among Chinese: isomers of C8 health project in China. Sci Total Environ. 2017;607-608:1304-12

10. Khalil N, Ebert JR, Honda M, Lee M, Nahhas RW, Koskela A, et al. Perfluoroalkyl substances, bone density, and cardio-metabolic risk factors in obese 8-12 year old children: a pilot study. Environ Res. 2018;160:314-21.

11. Ma S, Xu C, Ma J, Wang Z, Zhang Y, Shu Y, et al. Association between perfluoroalkyl substance concentrations and blood pressure in adolescents. Environ Pollut. 2019;254:112971.

12. Min J-Y, Lee K-J, Park J-B, Min K-B. Perfluorooctanoic acid exposure is associated with elevated homocysteine and hypertension in US adults. Occup Environ Med. 2012;69:658-62.

13. Yang Q, Guo X, Sun P, Chen Y, Zhang W, Gao A. Association of serum levels of perfluoroalkyl substances (PFASs) with the metabolic syndrome (MetS) in Chinese male adults: a cross-sectional study. Sci Total Environ. 2018;621: 1542-9.

14. Liao S, Yao W, Cheang I, Tang X, Yin T, Lu X, et al. Association between perfluoroalkyl acids and the prevalence of hypertension among US adults. Ecotoxicol Environ Saf. 2020;196:110589.

15. Chen A, Jandarov R, Zhou L, Calafat AM, Zhang G, Urbina EM, et al. Association of perfluoroalkyl substances exposure with cardiometabolic traits in an island population of the eastern Adriatic coast of Croatia. Sci Total Environ. 2019;683:29-36.

16. Christensen KY, Raymond M, Thompson BA, Anderson HA. Perfluoroalkyl substances in older male anglers in Wisconsin. Environ Int. 2016;91:312-8.

17. Donat-Vargas C, Bergdahl IA, Tornevi A, Wennberg M, Sommar J, Koponen J, et al. Associations between repeated measure of plasma perfluoroalkyl substances and cardiometabolic risk factors. Environ Int. 2019;124:58-65.

18. Geiger SD, Xiao J, Ducatman A, Frisbee S, Innes K, Shankar A. The association between PFOA, PFOS and serum lipid levels in adolescents. Chemosphere. 2014:98:78-83.

19. He X, Liu Y, Xu B, Gu L, Tang W. PFOA is associated with diabetes and metabolic alteration in US men: National Health and nutrition examination survey 2003-2012. Sci Total Environ. 2018;625:566-74.

20. Liu H-S, Wen L-L, Chu P-L, Lin C-Y. Association among total serum isomers of perfluorinated chemicals, glucose homeostasis, lipid profiles, serum protein and metabolic syndrome in adults: NHANES, 2013-2014. Environ Pollut. 2018;232:73-9.

21. Winquist A, Steenland K. Modeled PFOA exposure and coronary artery disease, hypertension, and high cholesterol in community and worker cohorts. Environ Health Perspect. 2014;122:1299-305.

22. Lin P-ID, Cardenas A, Hauser R, Gold DR, Kleinman KP, Hivert M-F, et al. Perand polyfluoroalkyl substances and blood lipid levels in pre-diabetic adults-longitudinal analysis of the diabetes prevention program outcomes study. Environ Int. 2019;129:343-53.

23. Williams B, Mancia G, Spiering W, Agabiti Rosei E, Azizi M, Burnier M, et al. 2018 ESC/ESH guidelines for the management of arterial hypertension. Eur Heart J. 2018:39:3021-104.

24. Duchon J. Splines minimizing rotation-invariant semi-norms in Sobolev spaces. In: Schempp W, Zeller K, editors. Constr Theory Funct Several Var [Internet]. Berlin, Heidelberg: Springer; 1977. [cited 2020 Apr 10]. p. 85-100. Available from: http://link.springer.com/10.1007/BFb0086566.

25. Wood S. Mgcv: Mixed GAM Computation Vehicle with GCV/AIC/REML Smoothness Estimation. 2012.

26. Levey AS, Stevens LA, Schmid CH, Zhang Y (Lucy), Castro AF, Feldman HI, et al. A New Equation to Estimate Glomerular Filtration Rate. Ann Intern Med. 2009;150:604.

27. Lewington S, Clarke R, Qizilbash N, Peto R, Collins R. Prospective studies collaboration. Age-specific relevance of usual blood pressure to vascular mortality: a meta-analysis of individual data for one million adults in 61 prospective studies. Lancet Lond Engl. 2002;360:1903-13.

28. Hardy ST, Loehr LR, Butler KR, Chakladar S, Chang PP, Folsom AR, et al. Reducing the Blood Pressure-Related Burden of Cardiovascular Disease: Impact of Achievable Improvements in Blood Pressure Prevention and Control. J Am Heart Assoc. 2015:4 [cited 2020 Jun 3]. Available from: https:// www.ahajournals.org/doi/10.1161/JAHA.115.002276.
29. Sundström J, Neovius M, Tynelius P, Rasmussen F. Association of blood pressure in late adolescence with subsequent mortality: cohort study of Swedish male conscripts. BMJ. 2011;342:d643.

30. Huang $M$, Jiao J, Zhuang $P$, Chen X, Wang J, Zhang Y. Serum polyfluoroalkyl chemicals are associated with risk of cardiovascular diseases in national US population. Environ Int. 2018;119:37-46.

31. Mastrantonio M, Bai E, Uccelli R, Cordiano V, Screpanti A, Crosignani P. Drinking water contamination from perfluoroalkyl substances (PFAS): an ecological mortality study in the Veneto region, Italy. Eur J Public Health. 2018;28:180-5.

32. Mattsson K, Rignell-Hydbom A, Holmberg S, Thelin A, Jönsson BA, Lindh CH, et al. Levels of perfluoroalkyl substances and risk of coronary heart disease: findings from a population-based longitudinal study. Environ Res. 2015;142: 148-54.

33. Simpson C, Winquist A, Lally C, Steenland K. Relation between perfluorooctanoic acid exposure and strokes in a large cohort living near a chemical plant. Environ Res. 2013;127:22-8.

34. Guzik TJ, Touyz RM. Oxidative stress, inflammation, and vascular aging in hypertension. Hypertension. 2017;70:660-7.

35. Lin C-Y, Lee H-L, Hwang $Y-T$, Su T-C. The association between total serum isomers of per- and polyfluoroalkyl substances, lipid profiles, and the DNA oxidative/nitrative stress biomarkers in middle-aged Taiwanese adults. Environ Res. 2020;182:109064.

36. Panaretakis T, Shabalina IG, Grandér D, Shoshan MC, DePierre JW. Reactive oxygen species and mitochondria mediate the induction of apoptosis in human hepatoma HepG2 cells by the rodent peroxisome proliferator and hepatocarcinogen, perfluorooctanoic acid. Toxicol Appl Pharmacol. 2001;173:56-64.

37. Wielsøe M, Long M, Ghisari M, Bonefeld-Jørgensen EC. Perfluoroalkylated substances (PFAS) affect oxidative stress biomarkers in vitro. Chemosphere. 2015;129:239-45.

38. Rodrigo R, González J, Paoletto F. The role of oxidative stress in the pathophysiology of hypertension. Hypertens Res. 2011;34:431-40.

39. Touyz RM, Rios FJ, Alves-Lopes R, Neves KB, Camargo LL, Montezano AC. Oxidative stress: a unifying paradigm in hypertension. Can J Cardiol. 2020; 36:659-70.

40. Rogers JM, Ellis-Hutchings RG, Grey BE, Zucker RM, Norwood J, Grace CE, et al. Elevated blood pressure in offspring of rats exposed to diverse chemicals during pregnancy. Toxicol Sci. 2014;137:436-46.

41. Borghi C, Urso R, Cicero AF. Renin-angiotensin system at the crossroad of hypertension and hypercholesterolemia. Nutr Metab Cardiovasc Dis. 2017; 27:115-20.

42. Kang JS, Choi J-S, Park J-W. Transcriptional changes in steroidogenesis by perfluoroalkyl acids (PFOA and PFOS) regulate the synthesis of sex hormones in H295R cells. Chemosphere. 2016;155:436-43.

43. Li Y, Fletcher T, Mucs D, Scott $K$, Lindh CH, Tallving P, et al. Half-lives of PFOS, PFHXS and PFOA after end of exposure to contaminated drinking water. Occup Environ Med. 2018;75:46-51.

44. Charkoudian N, Hart ECJ, Barnes JN, Joyner MJ. Autonomic control of body temperature and blood pressure: influences of female sex hormones. Clin Auton Res. 2017;27:149-55.

45. Kjeldsen LS, Bonefeld-Jørgensen EC. Perfluorinated compounds affect the function of sex hormone receptors. Environ Sci Pollut Res. 2013;20:8031-44.

46. Di Nisio A, Sabovic I, Valente U, Tescari S, Rocca MS, Guidolin D, et al. Endocrine disruption of androgenic activity by Perfluoroalkyl substances: clinical and experimental evidence. J Clin Endocrinol Metab. 2019;104:1259-71.

47. Di Nisio A, Rocca MS, Sabovic I, De Rocco PM, Corsini C, Guidolin D, et al. Perfluorooctanoic acid alters progesterone activity in human endometrial cells and induces reproductive alterations in young women. Chemosphere. 2020;242:125208.

48. Stergiou GS, O'Brien E, Myers M, Palatini P, Parati G. STRIDE BP: an international initiative for accurate blood pressure measurement. J Hypertens. 2020;38:395-9.

\section{Publisher's Note}

Springer Nature remains neutral with regard to jurisdictional claims in published maps and institutional affiliations. 